\title{
Analysis of the credit turnover in the "Retailer-Bank- Insurer" system with variations in market factors
}

\author{
M I Geraskin ${ }^{1}$, O A Kuznetsova ${ }^{1}$ \\ ${ }^{1}$ Samara National Research University, Moskovskoye shosse, 34A, Samara, Russia, 443086 \\ e-mail: olga_5@list.ru
}

\begin{abstract}
The article considers the possible states of the "Retailer-Bank-Insurer" system. The Pareto-efficient agents' strategies, which maximize the total profit of the system, are described. The agents' models parameters influence on the change in the state of the system is analyzed. Additionally, the influence of the complementarity effect on the change in the retailer's optimal sales volume is investigated. The values of the agents' parameters, which cause the transition of the system from one state to another are determined.
\end{abstract}

\section{Introduction}

The integrated organizational systems in the economy are formed in the event that the buyer's demand for one product is conditional upon the fact of purchasing another product. A typical example of such integration is the "retailer-bank-insurer" system, which is formed in the framework of the retailer's credit turnover. Hereinafter, the retailer, the bank, and the insurer are called the agents. The demand for retailer products encourages customers to apply for loans from banks and insurance services to insurers, and the possibility of obtaining credit resources, in turn, expands the demand for retailer products [1]. Therefore, the complementary demand leads to the integrated systems emergence [2].

The integrated system is tightly coupled [5], if the utility function (profit) of agent depends on its action and on the actions of the environment, i.e., other agents [3, 4]. In particular, the agents' costs of the "retailer-bank-insurer" system are interdependent (non-separable), since the system stability is ensured through mutual payments (commissions, discounts, etc.). The system agents revenues can be interdependent, if the system has a mechanism for the aggregate utility distribution [6, 7]. In this case, the agents' utilities can be considered as transferable.

As a result of the agents heterogeneity in economic activity, the problem of coordinating the agents' interests in the integration is considered. The agents, whose goods initiate demand for the goods of other agents, have prevailing economic activity. One of these agents is called the metaagent. The meta-agent status is realized in the awareness about other agents' utility functions and the utility values. The meta-agent chooses the distribution mechanism of the aggregated system utility. In the "retailer-bank-insurer" system the retailer is the meta-agent. The Pareto efficient [8] algorithm for the transferable utility distribution for the tightly coupled system was developed in [9].

In this paper, we use this algorithm to investigate the influence of the market environment parameters on the states of the "retailer-bank-insurer" system. Two types of states can be implemented in the system [10]. First, the meta-agent dominates, when the meta-agent's optimal sales volume is 
less than other agents' optimums; as a result, the environment agents reduce their volumes of sales to the meta-agent's volume. Second, the environment dominates, when the meta-agent's optimum exceeds other agents' optimums. Hence, there are two options of coordinating: either the meta-agent reduces sales to the volume of the environmental optimum level, or the environment increases sales to the meta-agent's optimum. The choice of these options is carried out in the system consistently [11, 12], according to the maximum of the total profit criterion. Consequently, the state of the system has a significant effect on the equilibrium after the agents' total profits distribution [13].

The subject of the paper is to study the influence of the agents' market demand functions parameters on the resulting system states and to determine the boundary values of these parameters, which cause transitions from one state to another.

\section{Methods and materials}

We consider the "retailer-bank-insurer" system. The utilities of agents are calculated by the following formulas [3]:

$$
\pi_{k}\left(Q_{k}\right)=\bar{p}_{k(n)} Q_{k}^{b_{k(n)}+1}-c_{k} Q_{k} k \in K,
$$

where $\bar{p}_{k}=a_{k}-u_{k}-p_{k}>0, k \in K ; \pi_{k}\left(Q_{k}\right)$ is the agent's profit function; $p_{k(n)}$ is the price of $k$-th agent's goods; $u_{k}$ is $k$-th agent's integration costs; $\rho_{k}$ is $k$-th agent risk; $a_{k(n)}, b_{k(n)}$ are the price function coefficients for the $n$-th system state $\left(n=1\right.$ is the integrated system with $Q_{-}{ }^{*}>Q_{l}^{*}, n=2$ is the integrated system with $Q_{-l i}{ }^{*}>Q_{l}{ }^{*}>Q_{-l j}{ }^{*}, n=3$ is the integrated system with $\left.Q_{-l}{ }^{*}<Q_{l}{ }^{*}\right) ; K$ is the agents' set; the meta-agent is denoted by the symbol «l», the environment is denoted by the symbol «$l »$, the symbol «*» denotes the optimum of the agent; $Q_{k}$ is $k$-th agent sales; $\rho_{k}$ is direct costs per unit of $k$-th agent.

The system states are classified as follows:

$$
n=\left\{\begin{array}{c}
1, \text { если } Q_{l}^{*}<Q_{-l}^{*}, \\
2, \text { если } Q_{l}^{*}>Q_{-l}^{*}, \\
3, \text { если } Q_{l}^{*} \in\left(Q_{-l 1}^{*}, Q_{-l 2}^{*}\right),
\end{array}\right.
$$

where the symbols $Q_{-l 1}^{*}, Q_{-l 2}^{*}$ denote the environmental agents of the meta-agent.

The meta-agent is an agent, which profit exceeds the total profit of the environment for the optimal strategy. The meta-agent is determined by the following criterion:

$$
\pi_{l}\left(Q_{l}^{*}\right)>\sum_{k \in K \backslash l} \pi_{k}\left(Q_{k}^{*}\right)
$$

The risk costs characterize the share of probable losses of the agent's average revenue from the price. The risk cost of the retailer is the bank's arrears on loans for goods sold. The bank's risk exposure is the overdue debt on loans issued, which is taken into account by the discount rate. The insurer's risk costs are payments for insured events, which are taken into account by the probability of their occurrence. In the case of $u_{k}>0$, the integration costs are interpreted as discounts or commissions in price of agent with greater economic activity. In the case of $u_{k}<0$ the integration costs represent the income of the more active agent as a transfer in the form of price premiums or commissions from other agents for participation in the integrated system.

The agents' optimums are calculated by the following formulas [3]:

$$
Q_{k}^{*}=\left[\frac{c_{k}}{\bar{p}_{k(n)}\left(b_{k}(n)+1\right)}\right]^{\frac{1}{b_{k}(n)}}, k \in K, n=1,2,3
$$

The complementary demand is taken into account as follows: the meta-agent sales volume through its demand function coefficient depends on the environment sales volume:

$$
\mathrm{a}_{1}=\mathrm{a}_{1 \mathrm{k}} \mathrm{a}_{10} \mathrm{Q}_{\mathrm{k}}^{*} \mathrm{k} \in \mathrm{K} \backslash \mathrm{l}, \mathrm{a}_{\mathrm{lk}}=\left\{\begin{array}{l}
\mathrm{a}_{\mathrm{lk}(1), \mathrm{n}=1,} \\
a_{\mathrm{lk}(2), \mathrm{n}=2,} \\
\mathrm{a}_{\mathrm{lk}(3), \mathrm{n}=3}
\end{array}\right.
$$

where $\alpha_{l k}$ is the complement ratio of $k$-th and $l$-th goods, $\alpha_{l k(1)}>\alpha_{l k(2)}>0$ are constants. The complementarity effect is expressed as follows: the growth in lending leads to a faster growth in 
retailer's turnover under low interest rate $(n=1)$, and it leads to a slow increase under high interest rate $(n=2)$.

The boundary values of the demand functions parameters are determined in the form of the following conditions:

$$
\begin{gathered}
Q_{i}^{*}\left(\widetilde{a}_{1}\right)=\min \left\{Q_{k}^{*}\left(\widetilde{a}_{k}\right), k \in K \backslash l\right\}, Q_{i}^{*}\left(\widetilde{a}_{i}\right)=\max \left\{Q_{k}^{*}\left(\widetilde{a}_{k}\right), k \in K \backslash l\right\}, \\
Q_{i}^{*}\left(\widetilde{b}_{1}\right)=\min \left\{Q_{k}^{*}\left(\tilde{b}_{k}\right), k \in K \backslash l\right\}, Q_{i}^{*}\left(\tilde{E}_{i}\right)=\max \left\{Q_{k}^{*}\left(\tilde{E}_{k}\right), k \in K \backslash l\right\},
\end{gathered}
$$

where $\tilde{a}_{k}, \tilde{a}_{k}, \tilde{b}_{k}, \tilde{b}_{k}, k \in K$ are the values of the demand functions coefficients under which the transition occurs between the states $n=1,2$ (denoted by the symbol " $\sim$ ") and $n=2,3$ (denoted by the symbol “ $\approx$ "). Because the system is tightly coupled, that is, the profits redistribution between agents is possible, the equilibrium is selected according to the agents' total profits criterion:

$$
\mathrm{Q}^{p}=\left\{Q_{k}^{p}, k \in K\right\}=\arg \max _{\mathrm{Q} \geq 0} \pi_{\Sigma}(\mathrm{Q}), \pi_{\Sigma}=\sum_{k \in K} \pi_{k}\left(Q_{k}^{*}\right)
$$

where $\mathrm{Q}^{p}$ is the Pareto-efficient (equilibrium) vector of the agents' actions $Q_{k}^{p} ; \pi_{\Sigma}$ is the total system profit.

We consider the problem of searching for the Pareto-effective strategies (i.e., actions) in the possible states of the "retailer-bank-insurer" system.

\section{Results}

Assertion 1. The Pareto-effective strategies of agents are as follows:

$$
\mathrm{Q}^{p}=\left\{Q_{k}^{p}, k \in K\right\}\left\{\begin{array}{c}
=Q_{l}^{*}, n=1, \\
\in\left[Q_{-l}^{*}, Q_{l}^{*}\right], n=2, \\
\in\left[Q_{-l 1}^{*}, Q_{-l 2}^{*}\right], n=3 .
\end{array}\right.
$$

Proof of assertion 1: for $n=1$, by criterion (6) we get $\mathrm{Q}^{p}=\left\{Q_{k}^{p}, k \in K\right\}=\arg \max _{\mathrm{Q} \geq 0}\left[\pi_{l}\left(Q_{l}^{*}\right)+\pi_{-l 1}\left(Q_{-l 1}^{*}\right)+\pi_{-l 2}\left(Q_{-l 2}^{*}\right)\right]$; because in this case, according to (2), $Q_{l}^{*}<Q_{-l}^{*}$, then, accounting (3), we can get $Q_{k}^{p}=Q_{l}^{*}, k \in K$. When $n=2$, by criterion (6) we get $Q_{-l}^{*} \leq Q_{k}^{p} \leq Q_{l}^{*}, k \in K$. When n $=3$, we get $Q_{-l 1}^{*} \leq Q_{k}^{p} \leq Q_{-l 2}^{*}, k \in K$.

In order to simulate the Pareto-effective strategies, we consider the "retailer-bank-insurer" system, in which the initiator of the coordination is the retailer. The parameters of the system are presented in Table 1.

Table 1. System parameters.

\begin{tabular}{llll}
\hline Parameters & Retailer & Bank & Insurer \\
\hline$a_{k}$ & 49000 & 0,785 & 0,35 \\
$u_{k}$ & -3230 & 0,031 & 0,018 \\
$\rho_{k}$ & 0 & 0,001 & 0,077 \\
$p_{k}$ & $49000 \mathrm{Q}_{1}{ }^{0,09}$ & $0,785 \mathrm{Q}_{2}{ }^{0,19}$ & $0,35 \mathrm{Q}_{3}{ }^{0,165}$ \\
$c_{k}$ & 1500 & 0,053 & 0,03 \\
$b_{k}$ & $-0,09$ & $-0,19$ & $-0,165$ \\
\hline
\end{tabular}

As follows from table 1, the maximum price of the retailer's product and its costs exceed the prices and costs of other agents, therefore, according to the maximum profit criterion, the retailer acts as a the meta-agent. The retailer receives additional income in the integration, because the integration costs of the retailer are negative.

In Figure 1, agent the profits dependence on sales volumes are plotted. It should be noted that due to the large difference in the prices of agents, the realtor's profit is estimated on the right scale, and the bank's profit and the insurer's profit are presented on the left scale. 


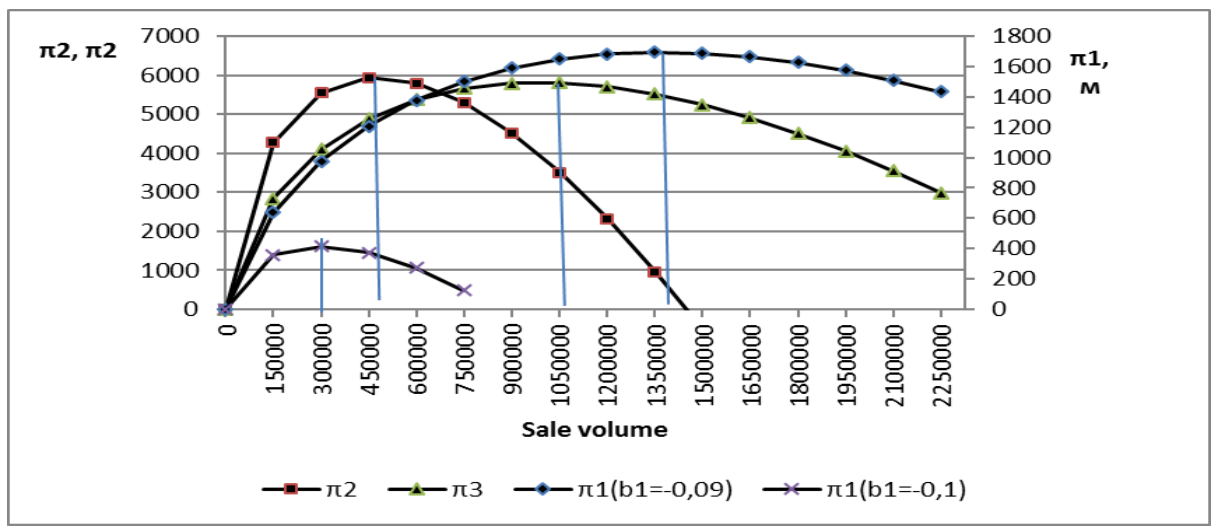

Figure 1. Dependence of agent profits on sale volumes.

In Figure 1, the following notation is used: $\pi_{1(b 1=-0,1)}$ is the retailer's profit, when its optimum is less than that of other agents; $\pi_{1(b 1=-0,09)}$ is the retailer's profit, when its optimum greater than that of other agents; $\pi_{2}$ is the bank's profit, $\pi_{3}$ is the insurer's profit.

The criterion for solving the problem is determined as the total profit of the system. In Figure 2, the total profit coincides with the retailer's profit due to its significant superiority over other agents. Thus, the total profit of the system is approximately equal to the retailer's profit. Consequently, the optimal sales volume of the system is determined by the retailer's model.

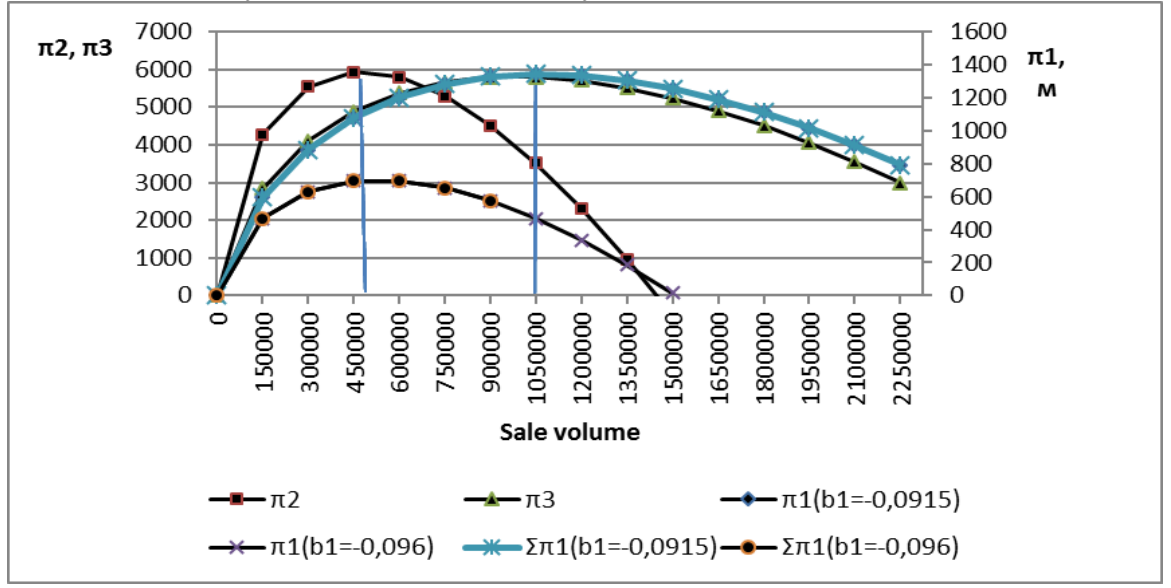

Figure 2. Dependencies of agent profits and total system profits.

In Figure 2, the following notation is used: $\pi_{1(b 1=-0,1)}$ is the retailer's profit, when its optimum less than that of other agents; $\pi_{1(b 1=-0,09)}$ is the retailer's profit, when its greater than that of other agents; $\pi_{2}$ is the bank's profit, $\pi_{3}$ is the insurer's profit, $\pi_{\Sigma 1(b 1=-0,0915)}$ is the total profit of non-integrated agents, when the optimal retailer's volume is greater than the optimal volumes of other agents, $\pi_{\Sigma 1(b 1=-0,096)} \mathrm{s}$ the total profit of non-integrated agents, when the optimal retailer's volume is less than the optimal volume of other agents.

The changing in the market parameters leads to a variety of system states, which is characterized by the position of $Q_{1}^{*}$ relative to other agents. In Figure 2, the set of the possible states reflects its boundary states, when $Q_{1(1)}{ }^{*}=Q_{2}{ }^{*}$ and $Q_{1(1)}{ }^{*}=Q_{3}{ }^{*}$. Accordingly, it is possible to distinguish three intervals: $Q_{1}{ }^{*}<Q_{2}{ }^{*}<Q_{3}{ }^{*}, Q_{2}{ }^{*}<Q_{1}{ }^{*}<Q_{3}{ }^{*}, Q_{2}{ }^{*}<Q_{3}{ }^{*}<Q_{1} *$.

Provided that the agent optimal volume depends on $b_{1}$, it is possible to determine the boundary values $b_{1}$. The situation $Q_{1}{ }^{*}=Q_{2} *<Q_{3} *$ is realized, when $b_{1}=-0,098$. The situation $Q_{2} *<Q_{3} *=Q_{1} *$ is reached, when $b_{1}=-0,098$. In the situation $\left(b_{1}=-0,1\right)$ the retailer's optimal volume $Q_{1(1)} *$ is less than the optimal volumes of other agents $Q_{2}{ }^{*}, Q_{3}{ }^{*}$. In the situation $\left(b_{1}=-0,09\right)$ the retailer's optimal volume $Q_{1(1)} *$ is greater than the optimal volumes of other agents $Q_{2}{ }^{*}, Q_{3}{ }^{*}$. Because the retailer's profit significantly exceeds other agents' profit, the total profit of agents in a non-integrated system 
almost coincides with the retailer's profit. Accordingly, it is possible to determine the optimum of the system as the optimum of the retailer.

We carry out the factor analysis of the market parameters influence $a_{1}, b_{1}, a_{2}, b_{2}$ on $Q_{1} *$.

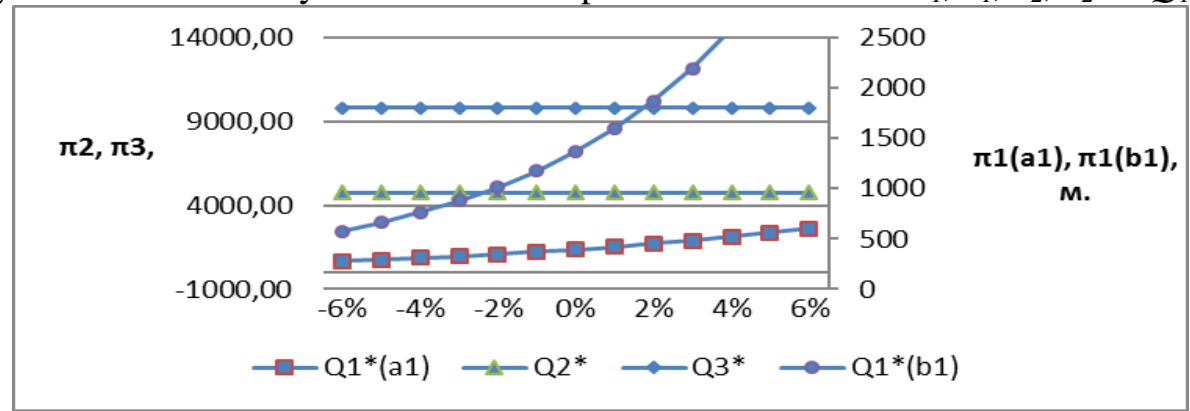

Figure 3. Dependencies of agents' optimal volumes on market factors $a_{1}, b_{1}$.

We consider the following initial market environment parameters : $a_{1}=49000, b_{1}=-0,019$. When changing the market parameters $a_{1}, b_{1}$, the complementarity effect does not arise, because the sales volumes of other agents do not change. Accordingly, the parameter $\alpha_{l k}$ in formula (5) is not taken into account. At the same time, it is obvious that an increase in $a_{1}$ is accompanied by a less sharp change in the retailer's optimal volume, than with a change in $b_{1}$. Thus, an increase in $a_{1}$ from 0 до $4 \%$ provides that it enters the zone $Q_{2}{ }^{*}<Q_{1} *<Q_{3}{ }^{*}$. An increasing in $a_{1}$ by more than $4 \%$ provides that the retailer's volume a switch to the zone $Q_{1}{ }^{*}>Q_{3}{ }^{*}$. At the same time, as $a_{1}$ decreases, the retailer's optimal volume decreases, and the situation $Q_{1} *<Q_{2} *$ arises.

An increasing in $b_{1}$ from $3 \%$ to $4 \%$ provides that the retailer's volume is in the zone $Q_{2}{ }^{*}<Q_{1}{ }^{*}<Q_{3}{ }^{*}$. An increasing in $b_{1}$ more than $4 \%$ provides that the retailer's volume is in the zone $Q_{1}{ }^{*}>Q_{3}{ }^{*}$. A decreasing in $b_{1}$ leads to the retailer's optimum reducing, and a situation $Q_{1} *<Q_{2} *$ arises.

When the market parameters $a_{2}, b_{2}$ change, the value of the bank's optimal volume changes, and the complementary effect arises that affects the retailer's sales. The calculation of the parameter $a_{1}$ by the formula (3) leads to an increase in $Q_{1}{ }^{*}$ with increasing $Q_{2}{ }^{*}$.

An increase in the $a_{2}$ leads to changes in the optimal volumes of the bank and the retailer. An increasing in $a_{2}$ from $-2 \%$ to $-1 \%$ leads to transition of the retailer's optimum in the zone $Q_{2} *<Q_{1} *<$ $Q_{3}{ }^{*}$. An increasing in $a_{2}$ from $-1 \%$ to $+\infty$ provides the retailer's volume transition to the zone $Q_{1}{ }^{*}>$ $Q_{3}{ }^{*}$. While decreasing in $a_{2}$ more than $2 \%$, the retailer's optimal volume is reduced, and a situation $Q_{1} *<Q_{2}^{*}$ arises.

Table 2. Characteristic intervals.

\begin{tabular}{llc}
\hline \multicolumn{1}{c}{$n=1$} & \multicolumn{1}{c}{$n=2$} & \multicolumn{1}{c}{$n=3$} \\
$\mathrm{Q} 1 *<\mathrm{Q} 2^{*}$ & \multicolumn{1}{c}{$\mathrm{Q} 2 *<\mathrm{Q} 1^{*}<\mathrm{Q} 3^{*}$} & $\mathrm{Q} 1^{*}>\mathrm{Q} 3^{*}$ \\
\hline$\Delta a_{1}(-\infty, 0 \%)$, & $\Delta a_{1}(0 \%,-4 \%)$, & $\Delta a_{1}(-4 \%,+\infty)$, \\
$\Delta b_{1}(-\infty, 3 \%)$ & $\Delta b_{1}(3 \%, 4 \%)$ & $\Delta b_{1}(4 \%,+\infty)$ \\
$\tilde{a}_{1}=49171_{x}, 5_{x}$ & & $\tilde{a}_{1}=54341_{x}$ \\
$\tilde{b}_{1}=-0,08964$ & & $\tilde{b}_{1}=-0,084$ \\
$\Delta a_{2}(-\infty,-2 \%)$, & $\Delta a_{2}(-2 \%,-1 \%)$, & $\Delta a_{2}(-1 \%,+\infty)$, \\
$\Delta b_{2}(-\infty,-1 \%)$ & $\Delta b_{2}(-1 \%, 1,6 \%)$ & $\Delta b_{2}(1,6 \%,+\infty)$ \\
$\tilde{a}_{2}=0,766_{x}$ & & $\tilde{a}_{2}=0,778_{x}$ \\
$\tilde{b}_{2}=-0,188$ & & $\tilde{b}_{2}=-0,0164$
\end{tabular}

A changing in $b_{2}$ from $-1 \%$ to $1,6 \%$ provides that the retailer's optimum is the zone $Q_{2}{ }^{*}<Q_{1} *<$ $Q_{3}{ }^{*}$. An increasing in $b_{2}$ more than $1,6 \%$ provides that the retailer's optimum switches to the zone $Q_{1}{ }^{*}>Q_{3}{ }^{*}$. When $b_{2}$ decreases more than $1 \%$, the retailer's optimal sales volume decreases and the situation $Q_{1} *<Q_{2}^{*}$ arises. 
The situation $\mathrm{n}=1$ denotes that the retailer's sales volume is in the interval $Q_{1} *<Q_{2}$. In this situation, the retailer's sales volume is the least in the system, hence, the retailer is interested in reducing the volume of other agents to $Q_{1}$

The situation $\mathrm{n}=2$ denotes that the retailer's sales volume is in the interval $Q_{2}{ }^{*}<Q_{1}{ }^{*}<Q_{3}{ }^{*}$. In this situation, the retailer's optimal volume is greater than the bank's optimum, but less than the insurer's optimum. The retailer encourages the bank to increase its sales, and it encourages the insurer to reduce its sales.

Situation $\mathrm{n}=3$ denotes that the retailer's sales volume is in the interval $Q_{1}{ }^{*}>Q_{3}{ }^{*}$. In this situation, the optimal sales volume of the retailer is greater than other agents' optimums, therefore, it is interested to encourage them to increase the sales.

The emergence of each situations is a result of a change in the parameters of the retailer: the maximum price of its product and the ratio of sales volume change to the price. Additionally, due to the complementarity effect, the change in bank's parameters through the coefficient of the demand function affects the change in the retailer's volume. Hence, the retailer's parameters are determined: the maximum price and the change in sales volume, when the transition from one situation to another occurs.

\section{Conclusion}

The paper considers the "retailer-bank-insurer" system. The system takes into account the integration costs and the joint influence of agents' sales volumes on each other. Several frontier positions have been defined, under which the Pareto-effective system strategy changes.

The zones $Q_{1}{ }^{*}<Q_{2}{ }^{*}, Q_{2}{ }^{*}<Q_{1}{ }^{*}<Q_{3}{ }^{*}, Q_{1}{ }^{*}>Q_{3}{ }^{*}$ are selected. In the first zone, the retailer, in order to increase its profit, is interested in encouraging the bank and the insurer to reduce their sales volumes to $Q_{1}{ }^{*}$. In the second zone, to achieve the same result, the bank must increase its sales volume, and the insurer must decrease. In the third zone, both the bank and the insurer must increase their sales to achieve $Q_{1}{ }^{*}$.

The influence of market factors $a_{k}, b_{k}$ on the optimal sales volume of agents is analyzed. An increase in these parameters leads to an increase in the optimal sales volumes of agents of the relevant market. At the same time, the change in the bank's parameters has an impact on the change in retailer's sales volume. The change in the retailer's optimum is more sensitive to changes in the bank's parameters.

\section{References}

[1] Kulikovskikh I M 2017 Anomaly detection in an ecological feature space to improve the accuracy of human activity identification in buildings Computer Optics 41(1) 126-133 DOI: 10.18287/2412-6179-2017-41-1-126-133

[2] Geraskin M I and Manakhov V V 2015 Optimization of interactions in a multi-agent, tightly linked "retailer-bank-insurer" system Problemy upravleniya 4 9-18

[3] Continuity and stimulating compatibility in cardinal voting mechanisms URL: https://papyrus. bib.umontreal.ca (15.07.2017)

[4] Novikov D A 2012 Theory of management of organizational systems

[5] Shao R and Zhou L 2016 Voting and optimal provision of public goods Journal of Social Economics 134 3-41

[6] Burkov V N, Gorgidze I I and Novikov D A 1997 Models and mechanisms for the distribution of costs and revenues in a market economy (Moscow: ICS RAS)

[7] Moulin H 2017 Designing a single mechanism Theoretical economics 12(2) 587-619

[8] Ventura A, Kafiero K and Montibeller M 2016 Pareto Efficiency, Coase's Theorem and External Effects: A Critical Look Journal of Economic Problems 50(3) 872-895

[9] Geraskin M I, Kuznetsova O 2017 Agents' Interaction algorithm in a strongly coupled system with a transferable utility CEUR Workshop Proceedings 1904

[10] Geraskin M I 2017 Optimal mechanism for the distribution of the effect in an integrated strongly coupled system of anonymous agents with a transferable utility Problemy upravleniya 2 27-41

[11] Pattanyk P K, Salles M 2016 Social choice and welfare (Elsevier science) 
[12] Bondarik V N, Kordin N A 2012 Resource allocation mechanisms based on non-maximum, symmetric, anonymous voting procedures with delegation Management problems 5 26-32

[13] Korgin N A, Korepanov V O 2016 Effective solution to the problem of resource allocation with the Groves-Lediar mechanism for portable utility Automation and remote control 77(5) 914-942 